\title{
Nondestructive Measurement of Nuclear Magnetization by Off-Resonant Faraday Rotation
}

\author{
R. Giri, ${ }^{1}$ S. Cronenberger, ${ }^{1}$ M. M. Glazov, ${ }^{2,3}$ K. V. Kavokin,,${ }^{2,3}$ A. Lemaître, ${ }^{4}$ J. Bloch, ${ }^{4}$ \\ M. Vladimirova, ${ }^{1, *}$ and D. Scalbert ${ }^{1}$ \\ ${ }^{1}$ Laboratoire Charles Coulomb UMR 5221 CNRS/UM2, Université Montpellier 2, \\ Place Eugene Bataillon, 34296 Montpellier Cedex 05, France \\ ${ }^{2}$ Ioffe Physical-Technical Institute of the RAS, 26, Politechnicheskaya, 194021 St. Petersburg, Russia \\ ${ }^{3}$ Spin Optics Laboratory, St. Petersburg State University, 1 Ul'anovskaya, Peterhof, St. Petersburg 198504, Russia \\ ${ }^{4}$ Laboratoire de Photonique et de Nanostructures, UPR CNRS, Route de Nozay, 91460 Marcoussis, France \\ (Received 12 March 2013; revised manuscript received 24 June 2013; published 20 August 2013)
}

\begin{abstract}
We report on the nondestructive measurement of nuclear magnetization in $n$-GaAs via cavity enhanced Faraday rotation. In contrast with the existing optical methods, this detection scheme does not require the presence of detrimental out-of-equilibrium electrons. Specific mechanisms of the Faraday rotation are identified for (i) nuclear spins situated within the localized electron orbits, these spins are characterized by fast dynamics, (ii) all other nuclear spins in the sample characterized by much slower dynamics. Our results suggest that even in degenerate semiconductors nuclear spin relaxation is limited by the presence of localized electron states and spin diffusion, rather than by Korringa mechanism.
\end{abstract}

DOI: 10.1103/PhysRevLett.111.087603

PACS numbers: 76.70.Hb, 71.70.Jp

Nuclear spin is currently considered as a promising system for quantum information processing [1-3]. It was shown that interaction between the light field and atomic ensembles allows for such phenomena as field-atom entanglement and spin squeezing [4,5]. In semiconductors, the challenge of the nuclear-spin memory has been seriously considered [1]. Powerful electrical, magnetic, and optical techniques were developed for nuclear spin detection [6-10]. Among them, optical techniques provide the best sensitivity, down to $10^{4}$ nuclei in a single quantum dot [10] and single nuclear spin of an impurity in diamond [2,11]. However, in most of the existing optical detection protocols, it is necessary to inject out-of-equilibrium carriers, in order to probe any changes in the electron spin polarization or splitting, induced by nuclear spin. Because injected electron spins are not in thermal equilibrium, they strongly affect nuclear spin dynamics, up to fueling spontaneous oscillations and giving rise to bistability $[6,10,12]$. Therefore, existing methods of optical detection never leave the nuclear polarization under study unperturbed.

In this Letter we experimentally realize a nondestructive, but sensitive and versatile, method of nuclear spin detection in semiconductors. It is based on off-resonant Faraday rotation (FR), a phenomenon in which the polarization of a light field rotates depending on the spin polarization in an optical medium. Nuclear spin detection by FR has been demonstrated in atomic vapors [13], but in semiconductors the detection of nuclear spin has only been achieved under optical pumping. In this case, large FR is induced by optically polarized electrons due to Pauli blocking in the conduction band, as illustrated in Fig. 1(a). This property is exploited in optical detection of nuclear magnetic resonance (NMR) by Larmor magnetometry [7]. In the absence of optical pumping, such experiments are not possible. In this case, nuclear spin polarization can only be detected by virtue of either the conduction level spin splitting or the equilibrium electron spin polarization induced by the effective nuclear (Overhauser) field $B_{N}$. To selectively probe this weak nuclear FR we use a two-step protocol, proposed by Artemova and Merkulov [14]. In the first step, nuclei are
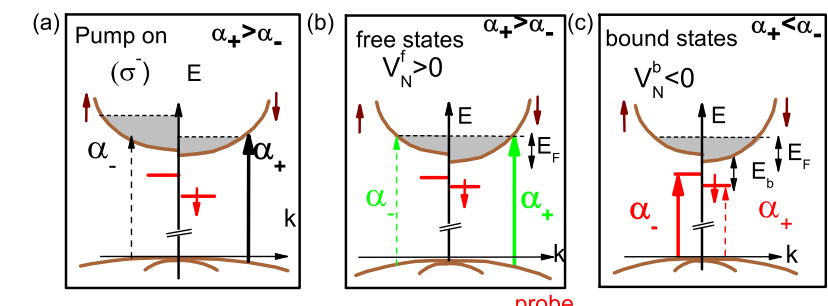

(d)
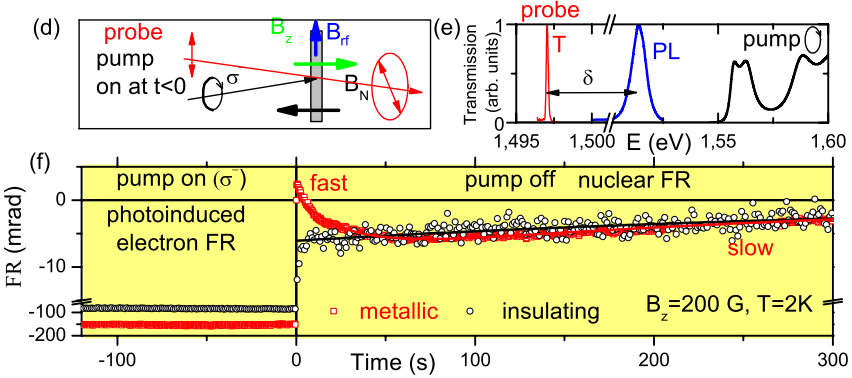

FIG. 1 (color online). (a)-(c) Band diagrams with relative absorption probabilities $\alpha_{+}, \alpha_{-}$for the two opposite light helicities: under $\sigma^{-}$optical pumping (a) and in the presence of nuclear field only, for the transitions involving free (b) and bound (c) electron states. For clarity, only transitions from the heavy hole band are shown. (d) Sketch of the experimental setup. (e) Calculated transmission of the microcavity sample above the stop band (black), measured transmission of the probe beam (red), and photoluminescence (PL, blue). (f) FR scans for two samples, including preparation $(t<0)$ and measurement $(t>0)$ stage. Total pumping time is $10 \mathrm{~min}$. 
dynamically polarized by conventional optical pumping in the presence of a longitudinal magnetic field. Then, optical pumping is switched off. The electron spin system returns to equilibrium within several hundreds of nanoseconds $[15,16]$. In contrast, nuclear spin polarization "in the dark" can last for minutes $[10,17]$. We show that it generates measurable FR via dispersive response of conduction band states in the presence of effective nuclear field. To increase the detection sensitivity, we designed the sample as a planar microcavity with the Fabry-Perot resonance corresponding to the probe energy.

This scheme is applied here to $n$-GaAs. Complex dynamics of nuclear spins is revealed in this well-studied material. We show that nuclear FR is not only measurable, but is also sensitive to both (i) nuclear spins characterized by fast relaxation and situated within the localized electron orbits and (ii) all other nuclear spins in the sample, characterized by much slower relaxation. Depending on the position, the dominant microscopic mechanisms of nuclear FR are different, as sketched in Figs. 1(b) and 1(c). Electron spin polarization induced by nuclear field dominates in case (i); it favors absorption at the highest spin state due to Pauli exclusion principle. In contrast, in case (ii) conduction band splitting favors absorption at the lowest spin band, where higher density of states is available. Thus, circular dichroism $\left(\alpha_{+}-\alpha_{-}\right)$has opposite sign in these two cases, providing spatial selectivity of the FR and giving clues for understanding complex nuclear spin relaxation in metallic $n$-GaAs [8]. NMR related to the magnetization of different nuclear species is also detected by FR.

The studied structures consist of a Si-doped GaAs $3 \lambda / 2$ cavity with electron concentrations $n_{e}=2 \times 10^{16} \mathrm{~cm}^{-3}$ (metallic) and $n_{e}=2 \times 10^{15} \mathrm{~cm}^{-3}$ (insulating). The front (back) mirrors are distributed Bragg reflectors composed of 25 (30) pairs of $\mathrm{AlAs} / \mathrm{Al}_{0.1} \mathrm{Ga}_{0.9}$ As layers, grown on a $400 \mu \mathrm{m}$ thick GaAs substrate. Because of multiple round trips in the cavity, the FR is amplified by a factor $N \sim 1000$, corresponding to the interaction length $L=0.7 \mathrm{~mm}$. The sample was placed in a cryostat $(T=2$ or $4 \mathrm{~K})$ surrounded by resistive coils for Earth field compensation, and for application of static and rf magnetic fields [Fig. 1(d)]. During the preparation stage $(t<0)$, a circularly polarized pump beam at $E_{p p}=1.59 \mathrm{eV}$ is focused onto a $500 \mu \mathrm{m}$ spot on the sample surface. It polarizes the electron gas in the cavity [16]. In order to meet the conditions for dynamic nuclear polarization, a longitudinal magnetic field (typically $B_{z}=200 \mathrm{G}$ ) is applied [18]. To probe FR with the optimum stability, a spectrally broad $(20 \mathrm{meV})$ linearly polarized pulse provided by a mode-locked Ti-sapphire laser is used. It is centered at the cavity mode, which filters the incident pulse at $E_{p r}=1.497 \mathrm{eV}$, corresponding to the detuning $\delta=18 \mathrm{meV}$, with respect to the photoluminescence maximum; see Fig. 1(e). Typical pump and probe powers are 10 and $2 \mathrm{~mW}$, respectively. The measurement stage starts as soon as the pump is switched off and electron spin polarization is returned to equilibrium $(t \approx 250 \mathrm{~ns})$ [16]. Most of the data presented below correspond to the metallic sample. Static FR caused by the external magnetic field is systematically subtracted from raw data, except for the experiments under alternating field.

Figure 1(f) shows typical FR scans. Under optical pumping $(t<0)$ a strong FR signal is generated by optically polarized electron spin. Its sign is determined by the pump helicity, and its microscopic origin resides in the Pauli blocking, as shown in Fig. 1(a) [19]. Stronger FR in the metallic sample is due to longer electron spin relaxation time. As soon as the pump beam is switched off, the signal drops on the scale of electron spin relaxation time. The remaining FR is much weaker, and is only due to spin polarized nuclei. Surprisingly, while in the insulating sample a monotonic decay of the signal associated with nuclear spin relaxation is observed, a nonmonotonic behavior is clearly seen in the metallic sample. In fact, in the metallic sample there are two contributions to the FR, with strongly different decay times, comparable amplitudes, and opposite signs. The characteristic decay times of the "fast" and "slow" components are of the order of several seconds and several hundred seconds, respectively.

Figure 2(a) shows the effect of pumping duration and helicity on the nuclear FR in the metallic sample.
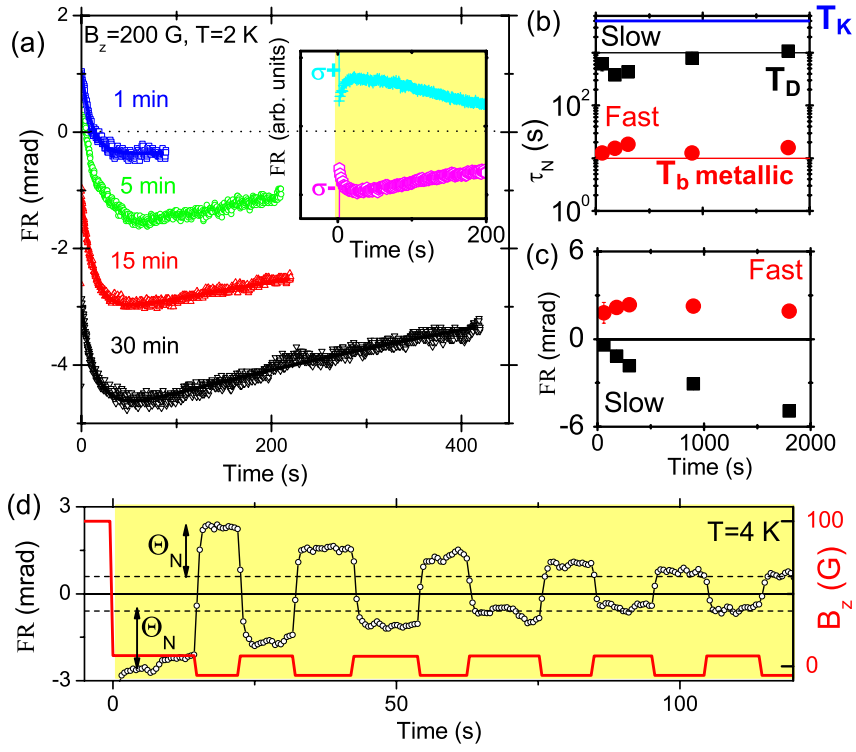

FIG. 2 (color online). (a) Faraday rotation induced by nuclear polarization prepared by $\sigma^{-}$pumping at $B_{z}=200 \mathrm{G}$. Solid lines are fits to the biexponential decay. Inset: Effect of pump helicity on the nuclear FR. Decay times (b) and amplitudes (c) deduced from biexponential fit of the data shown in (a). Solid lines are calculated nuclear spin relaxation times. $T_{b}$, nuclei under the bound electron orbit; $T_{D}$, diffusion-limited relaxation; $T_{K}$, Korringa relaxation. (d) Nuclear repolarization experiment. Preparation during $6 \mathrm{~min}$., $\sigma^{-}$pumping, $B_{z}=100 \mathrm{G}$. At $t=0$ the pump is switched off and alternating field is applied $( \pm 7 \mathrm{G}$, right-hand scale). Static FR in the external field $B_{z}= \pm 7 \mathrm{G}$ is shown by dashed lines. 
The nonmonotonic behavior is systematic. The sign of both components can be controlled either by the pump helicity (Fig. 2, inset) or by reversing the magnetic field direction after the preparation stage. This behavior is consistent with the nuclear spin origin of the FR [20]. The corresponding decay times and amplitudes are reported in Figs. 2(b) and 2(c). The decay times for the two components do not depend on the pumping time and always differ by a factor of $\sim 50$. Figure 2(c) shows that the buildup times are correlated with the decay times.

In order to ascertain the relevant microscopic mechanisms, it is important to measure the sensitivity of the nuclear FR. It can be defined in terms of the nuclear Verdet constant $V_{N}$. In analogy with the traditional Verdet constant ( $V<0$ under the same conditions), this quantity is defined from $\Theta_{N}=V_{N} L B_{N}$. The nuclear field $B_{N}$ corresponding to given cooling conditions is determined from independent experiments for the metallic sample [21]. We obtained $V_{N}=0.1 \mathrm{mrad} /(\mathrm{cm} \mathrm{G})$ for the slow FR component, while it was not possible to determine the Verdet constant associated with the fast component. We have also verified that no photoinduced effects show up when pumping the substrate in the same conditions, which definitively excludes any contribution from the substrate in the observed phenomena.

The first key to understanding the complex pattern of time-dependent nuclear spin induced FR is the time scales of its slow and fast components. At few-K temperatures, nuclear spin-lattice relaxation is entirely due to interaction with electron spins [23]. The question is, can the slow and fast relaxations be due to the presence of both mobile (free) and bound electron states in the sample? In a degenerate electron gas, single donors do not bind electrons because of the screening. Therefore, the density of shallow bound states is determined by the concentration of closely spaced donor pairs $N_{b}$ [24]. It can be estimated as $N_{b} \approx$ $2 \times 10^{15} \mathrm{~cm}^{-3}[24,25]$. Bound electrons provide an efficient source of relaxation for the nuclei, situated under their orbit, $T_{b}=1 /\left(\Omega_{N}^{2} \tau_{c}\right) \approx 10 \mathrm{~s}$, consistent with the fast component relaxation time [26]. Here, $\Omega_{N}$ is nuclear spin precession frequency in the instantaneous Knight field $B_{e} \approx 100 \mathrm{G}$ created by the localized electron [27], and $\tau_{c} \approx 10^{-11} \mathrm{~s}$ is the correlation time of the localized electron spin, determined by exchange scattering with free electrons $[28,29]$. Thus, the fast component can be associated with the nuclei situated under the orbits of localized electrons. Similar estimations for the insulating sample yield $T_{b} \approx 0.1 \mathrm{~s}$, due to much longer correlation time $\tau_{c} \approx 3 \times 10^{-10} \mathrm{~s}$ [15]. This is beyond the temporal resolution of our experiment, so the fast component could not be detected in the insulating sample.

The slow component of the FR can be related to the nuclei remote from the localized centers. Indeed, under optical pumping these remote nuclei can get polarized by spin diffusion from localized centers [17], or by dynamic nuclear polarization involving free electrons [30]. These processes are much slower than dynamic nuclear polarization by localized electrons. Thus, the pumping time required for the buildup of the slow component is much longer, consistent with our observations. The spin relaxation of the remote nuclei in the metallic sample is expected to be induced by flip flops with the electrons from the thermally broaden Fermi edge. The corresponding time is called Korringa relaxation time, $T_{K}[9,23]$. At $T=2 \mathrm{~K}$ it can be estimated as $T_{K} \approx 10^{4} \mathrm{~s}$, which is much longer than the slow component decay time. We suggest that nuclear relaxation is dominated by the nuclear spin diffusion towards the donor pairs. The time of this diffusion-limited relaxation can be estimated as [31] $T_{D}^{-1} \approx 4 \pi D N_{b} a$, where $D \approx 10^{-13} \mathrm{~cm}^{2} / \mathrm{s}$ is the nuclear spin diffusion coefficient [17] and $a$ is the localization radius of the electron on the center. Assuming $a=10 \mathrm{~nm}$, we obtain $T_{D} \approx$ $10^{3} \mathrm{~s}$, close to the values measured for the slow component relaxation. In the insulating sample, the donor concentration is of the same order as the concentration of donor pairs $N_{b}$ in the metallic sample. Thus we expect here the diffusion-limited spin relaxation on the same time scale, consistent with the experimental observations [Fig. 1(f)]. Thus, nuclear spin relaxation in both samples is determined by spin diffusion towards the regions in the sample, where efficient relaxation involving localized electrons takes place [32].

The most intriguing experimental fact is the sign difference between the two components of nuclear FR. It can be understood in terms of the underlying microscopic mechanisms, as illustrated in Figs. 1(b) and 1(c). The nuclear field splits both conduction-band states and bound electron states, and polarizes the electrons. In our experiments the detuning is large enough $\delta \gg E_{F}, \delta \gg E_{b}$, where $E_{b}$ is the localized electron binding energy and $E_{F}$ is Fermi energy. Under these conditions, the contribution of the free states in the circular dichroism and birefringence is mainly due to the spin splitting: the absorption at lower spin state is favored, since larger density of states is available. In contrast, the contribution of the bound states is dominated by the Pauli blocking, so that a higher energy spin state provides stronger absorption. This brings forth the sign difference for the circular dichroism $\left(\alpha_{-}-\alpha_{+}\right)$in these two situations; see Figs. 1(b) and 1(c).

Quantitatively, the contributions to the FR from free and bound electron states can be given in terms of the corresponding Verdet constants [14,19,33]:

$$
\begin{gathered}
V_{N}^{f}=-\frac{e^{2} \hbar}{12 m c n} \frac{g_{e} \mu_{B}}{4 \sqrt{\delta}} \sum_{h=h h, l h}\left(\frac{2 \mu_{h}}{\hbar^{2}}\right)^{3 / 2}, \\
V_{N}^{b}=\frac{\pi \hbar^{3} c^{2}}{E_{0}^{2} \tau_{r} n} \frac{N_{b} g_{e} \mu_{B}}{4 k_{B} T \delta} .
\end{gathered}
$$

Here, $c$ is the speed of light, $m$ is the electron effective mass, $\mu_{h h}\left(\mu_{l h}\right)$ are the reduced masses of the 